\section{Cardialagia \\ PERFORMANCE DIAGNÓSTICA DE ANGIOGRAFIA CORONARIANA POR TOMOGRAFIA COMPUTADORIZADA DE 64 DETECTORES (ESTUDO CORE 64)}

A angiografia coronariana convencional (ACC) permanece atualmente como o exame padrão-ouro para avaliação de doença arterial coronária (DAC), pois permite determinar a extensão, localização e gravidade das lesões obstrutivas coronarianas. A angiografia coronariana por tomografia computadorizada (ACTC) de múltiplos detectores tem sido utilizada como um teste nãoinvasivo para avaliação da presença de obstrução coronariana, porém a acurácia deste método ainda não é bem estabelecida. Recentemente, foram publicados dois estudos multicêntricos que procuraram avaliar a acurácia diagnóstica desta metodologia.

O estudo CORE 64 foi desenhado para determinar a acurácia de angiografia coronariana por TC de 64 detectores comparada à angiografia coronariana convencional em pacientes com suspeita de doença arterial coronária. Miller J. $\mathrm{M}^{1}$ et al. conduziram este estudo internacional, multicêntrico e prospectivo, envolvendo nove hospitais em sete países. Foram incluídos pacientes acima de 40 anos, com suspeita clínica de DAC sintomática e todos com indicação de realização de angiografia coronariana convencional. Todos pacientes realizaram os exames de escore de cálcio e angiografia por TC de 64 detectores antes da angiografia convencional. Os exames de ACTC e ACC foram analisados em laboratórios independentes e as estenoses acima de 50\% foram consideradas como lesões obstrutivas. Foram excluídos da análise os pacientes com escores de cálcio superiores a 600 (Agatston). Dos 291 pacientes incluídos na análise, 56\% apresentaram doença coronária obstrutiva. A acurácia diagnóstica da angiografia por TC para detectar lesões acima de 50\% quando comparada à angiografia convencional obteve uma área sob curva ROC (receiver-operating-characteristic) de 0,93 (IC 95\%, 0,90 a 0,96). A sensibilidade do método foi de 85\% (IC 95\%, 79 a 90), especificidade de 90\% (IC 95\%, 83 a 94), valor preditivo positivo de $91 \%$ (IC 95\%, 86 a 95) e valor preditivo negativo de $83 \%$ (IC 95\%, 75 a 89). Os autores concluíram que a angiografia coronariana por TC de múltiplos detectores apresenta boa acurácia para identificar a presença e gravidade de doença arterial coronária obstrutiva, porém os valores preditivos positivos e negativos indicam que este método ainda não substitui a angiografia coronariana convencional.

Num trabalho semelhante, Meijboom W.B. ${ }^{2}$ et al. propuseram estudar a acurácia diagnóstica de angiografia coronariana por TC de 64 detectores para detectar ou excluir DAC significativa. Pacientes sintomáticos com diagnósticos estabelecidos de angina estável ou angina instável, com indicação clínica de realização de angiografia coronariana convencional, foram incluídos neste estudo multicêntrico, de forma prospectiva, envolvendo três hospitais universitários. Foram incluídos na análise 360 pacientes entre 50 e 70 anos que realizaram as duas angiografias coronárias: a convencional e a por TC. O diagnóstico da doença arterial coronária foi baseado na presença de obstruções coronarianas $\geq 50 \%$. Neste estudo, todos os vasos e segmentos coronarianos foram incluídos na análise, independentemente da qualidade de imagem obtida. A prevalência de DAC foi de $68 \%$ neste estudo. A sensibilidade de ACTC para detecção de lesões obstrutivas foi de 99\% ( IC 95\%, 98 a 100\%), especificidade de 64\% (IC 95\%, 55 a 73\%), valor preditivo positivo de 86\% (IC 95\%, 82 a $90 \%$ ) e valor preditivo negativo de 97\% (IC 95\%, 94 a100\%). Os autores ressaltam que a angiografia coronariana por TC pode excluir DAC significativa em pacientes já com indicação de angiografia coronariana convencional. Além disso, observaram que a gravidade das lesões ateroscleróticas geralmente é superestimada por angiografia coronaria por TC e concluíram que são necessários estudos futuros para melhor definição do manejo terapêutico dos pacientes com os resultados deste exame.

\section{Comentário}

A angiografia coronariana por tomografia computadorizada de múltiplos detectores emergiu nos últimos anos como um exame promissor para substituição de angiografia coronariana convencional. Desde seu advento, este exame despertou grande interesse e entusiasmo no meio médico por ser um teste simples, elegante e não-invasivo que permite detectar a presença de obstrução coronariana. A ACTC passou a ser amplamente utilizada para avaliação cardiológica de pacientes com suspeita de doença arterial coronária, apesar da carência inicial de evidência científica acerca do método, indefinição da sua acurácia diagnóstica e da sua aplicabilidade clínica.

Os primeiros estudos publicados com ACTC obtidos por meio de tomógrafo de 4, 8 e 16 detectores mostravam limitações importantes do método devido à baixa nitidez das imagens adquiridas e dificuldades na análise dos segmentos coronarianos distais. A melhora de aquisição de imagem obtida com o advento de tomógrafo de 64 detectores possibilitou uma melhora na análise de todo território coronariano, incluindo os segmentos coronarianos médios e distais e resultou num grande crescimento na utilização deste exame na prática clínica. Revisão sistemática ${ }^{3}$ dos estudos publicados nos últimos anos mostrou uma melhora da sensibilidade e especificidade da ACTC, sobretudo no seu valor preditivo negativo elevado (próximo a 100\%), tornando este um exame interessante para exclusão de doença arterial coronária e tem sido advogado como o exame que veio para substituir a angiografia coronariana convencional. Entretanto, devemos lembrar que os estudos apresentados até então eram estudos pequenos, de um único centro, envolvendo pacientes selecionados e, frequentemente, os segmentos com baixa nitidez da imagem eram excluídos da análise.

Estes dois estudos multicêntricos publicados recentemente mostram que a angiografia coronariana por TC de múltiplos detectores apresenta uma acurácia diagnóstica relativamente boa para detecção ou exclusão das lesões obstrutivas acima de $50 \%$ em pacientes com risco intermediário a alto de DAC. Pórem, diferentemente do que vem sendo mostrado nos estudos prévios, apresenta um valor preditivo negativo longe do ideal (83\%) quando comparado com o exame padrão-ouro que continua sendo a angiografia coronariana convencional. A baixa 
especificidade do método e o número de exames falso-positivos observados no estudo de Meijboom, quando faz a análise de todos segmentos estudados, é bastante preocupante e nos mostra a necessidade de cautela. A positividade da ACTC geralmente acarreta uma série de outros testes complementares ou procedimentos adicionais, e consequente elevação de custos sem exato conhecimento do seu benefício e sua implicação no acompanhamento clínico dos pacientes.

A angiografia coronariana por TC ainda apresenta limitações técnicas que precisam ser aprimoradas, tais como a dificuldade na análise de placas muito calcificadas e de segmentos muito distais, além do impacto que a dose de radiação poderia causar nos pacientes ser desconhecido. O seu uso indiscriminado não está justificado e acarreta o aumento de custo no sistema de saúde, sem evidências científicas claras do seu benefício. Ainda faltam estudos que demonstrem o custo-efetividade relacionado a esta nova tecnologia, a determinação do perfil dos pacientes que poderiam se beneficiar do método e principalmente o efeito prognóstico na evolução dos pacientes. A sua utilização como método para avaliação de doença arterial coronária ainda precisa ser melhor definida com estudos futuros.

PaI ChIng Yu*

Bruno Caramelli Daniela Calderaro

\section{*Correspondência:}

Av. Dr. Enéas de Carvalho Aguiar, 44 andar AB Bloco II Unidade clínica de Medicina Interdisciplinar em Cardiologia CEP: 01546-020

São Paulo - SP

Referências

1. Miller JM, Rochitte CE, Dewey M, Arbab-Zadeh A, Nunuma H, Gottlieb I, et al. Diagnostic performance of coronary angiography by 64-Row CT. N Engl J Med. 2008;359(22):2324-36.

2. Meijboom WB, Meijs MFL, Schuijf JD, Cramer MJ, Mollet NR, Van Mieghem CA, et al. Diagnostic accuracy of 64-slice computed tomography coronary angiography. J Am Coll Cardiol. 2008;52(25):2135-44.

3. Stein PD, Yaekoub AY, Matta F, Sostman HD. 64-Slice CT for diagnostic of coronary artery disease: a systematic review. Am J Med. 2008;121(8):715-25.

\section{Ginecolagia \\ OOFORECTOMIA PROFILÁTICA NA HISTERECTOMIA POR AGRAVO UTERINO BENIGNO: EVIDÊNCIAS ATUAIS}

A ooforectomia profilática durante uma histerectomia por agravo uterino benigno representa tema controverso. A prática concomitante desses dois procedimentos atinge mais de 300 mil casos anuais nos EUA e, 50\% deles são realizados em mulheres com idade entre 40 e 64 anos, quando os ovários ainda exibem significativa atividade endócrina.

De fato, ao redor de 37 anos constata-se nítida claudicação hormonal dos ovários, quando sua população folicular é de 20 mil unidades; a partir daí, instalam-se pequenas oscilações na produção dos esteróides, justificando as primeiras irregularidades menstruais características do estágio da transição menopausal.

Quando o número de folículos alcança 1.000 unidades, os ovários cessam definitivamente a produção de esteróides, resultando na última menstruação da vida da mulher - a menopausa - emergindo, a partir de então, o estágio da pós-menopausa.

Apesar da evidente escassez de folículos após a menopausa, os ovários ainda mantêm normal a síntese de androgênios (testosterona e androstenediona) no seu estroma, que transportados ao tecido adiposo periférico se convertem em estrona, o principal estrogênio da pós-menopausa.

Assim, após uma ooforectomia, com a queda na produção dos androgênios, ocorre redução de 40\%-50\% nas concentrações séricas de estrona, explicando o aparecimento de sintomas e sérios agravos. As ondas de calor constituem os sintomas mais desconfortáveis e acometem 90\% das mulheres ooforectomizadas, diferentemente da prevalência de 50\% observada na população feminina, quando a menopausa se instala espontaneamente. Sintomas psíquicos e sexuais também são referidos e decorrem da redução de $40 \%$ na produção androgênica.

No estágio da pós-menopausa com a evidente deficiência de estrogênios podem eclodir importantes agravos como atrofia cutâneo-mucosa, doença cardiovascular, osteoporose e distúrbios da cognição ${ }^{1}$.

Os mecanismos envolvidos no maior risco cardiovascular observado em mulheres ooforectomizadas ainda não estão bem esclarecidos; admite-se como o mais possível a perda dos efeitos benéficos dos estrogênios sobre o metabolismo lipídico e endotélio vascular.

Nesse sentido, as evidências mostram o dobro do risco de infarto agudo do miocárdio nas mulheres ooforectomizadas entre 40-44 anos e incremento de 40\% naquelas com 50-59 anos; entretanto, o risco não foi maior quando o ato cirúrgico ocorreu após os 65 anos $^{3}$.

Nesse sentido, Dorum et al. ${ }^{4}$ em estudo populacional envolvendo mulheres entre 40 e 69 anos observaram que a ooforectomia bilateral associada ou não a histerectomia redunda num maior risco de síndrome metabólica e, consequentemente da DCV e do diabetes tipo 2.

A ooforectomia também acarreta expressivas repercussões para a massa óssea, visto que os estrogênios fisiologicamente exercem ação antirreabsortiva e os androgênios, além daquele efeito, ainda atuam na formação óssea. Estudos em mulheres com ooforectomia, acompanhadas por mais de 16 anos, revelaram não só maior prevalência de fraturas osteoporóticas, mas também aumento da mortalidade após pequenos traumas naquelas com fraturas de quadril aos 60 anos ${ }^{5}$. Ademais, a preservação dos ovários promove ainda redução de $50 \%$ na mortalidade por todas as causas ${ }^{6}$.

Contrastando aos riscos apontados, as evidências mostram que a ooforectomia profilática pode redundar em benefícios, como a prevenção dos cânceres de mama/ovário e a redução do número de futuras ooforectomias. No tocante ao câncer de ovário, evidências epidemiológicas e ilações matemáticas sugerem uma 